\title{
Restoring Vision Using Stem Cells and Transplantation
}

\author{
Elisa Cuevas, Paresh Parmar and Jane C. Sowden
}

Stem Cells and Regenerative Medicine Section, University College London Great Ormond Street Institute of Child Health, and NIHR Great Ormond Street Hospital Biomedical Research Centre, 30 Guilford Street, London, WC1N 1EH, UK, j.sowden@ucl.ac.uk.

\begin{abstract}
The replacement of retinal cells, or the support of surviving retinal neurons, in a degenerated retina presents a significant challenge in the fields of ophthalmology and regenerative medicine. Stem cell-based therapies are being explored as an approach for treating retinal dystrophies, such as retinitis pigmentosa (RP), Stargardt's disease, and age-related macular degeneration (AMD). This review provides an update on the recent progress made toward the restoration of vision lost to degenerative disease using stem cell-based transplantation strategies and the challenges that need to be overcome. Both retinal pigmented epithelium (RPE) and photoreceptor replacement therapies are discussed.
\end{abstract}

\section{Keywords}

Cell therapy, Cell transplantation, Retinal degeneration, Retinitis pigmentosa, Age-related macular disease, Stem cell therapy, Retinal organoids, Translational research, In vitro retinal pigmented epithelium, In vitro photoreceptor cells 


\subsection{Introduction}

In the UK alone, more than two million people suffer from partial or complete blindness (Pezzullo et al. 2018). Approximately half are affected by AMD, a pathology that causes the loss of photoreceptors preceded by damage to the RPE in the central retina (the macula). In addition, mutations in more than 200 genes cause inherited retinal dystrophies, including RP, Stargardt's disease, and Leber's congenital amaurosis. Gene replacement therapy clinical trials have shown good outcomes by correcting deficient gene expression in surviving cells (Bainbridge et al. 2015). For patients at an advanced stage of disease, with significant degeneration of photoreceptor cells in the outer nuclear layer (ONL) and/or loss of macular cell populations, transplantation represents an opportunity to replace the lost cells with new functional cells and may also act to preserve cells or augment the function of abnormal cells.

While lower vertebrates, such as zebrafish, possess a capacity to self-regenerate the retina (Powell et al. 2016), such a mechanism is not apparent in mammals. Therefore, cell replacement has been investigated using mammalian preclinical models. Proof-of-concept studies identified the optimal age of donor photoreceptors, derived from immature murine primary retinal tissue, to contribute toward sight recovery (MacLaren et al. 2006; Pearson et al. 2012; Singh et al. 2013). Pluripotent stem cells (PSCs) hold many advantages over primary retinal cells. Able to selfrenew and differentiate into nearly every cell type in the body including retinal cells, they have the potential to provide an unlimited source of cells that could be used to repair the degenerated retina, regardless of the genetic cause of the patient's condition.

Several promising strategies for RPE and photoreceptor replacement that utilize PSCs are under investigation for AMD and inherited retinal dystrophies, respectively. They rely on the use of 
embryonic stem cells (ESCs) or somatic cell reprogramming to generate induced PSCs (iPSCs). In each case, several critical steps are necessary to establish a clinically relevant therapy: (i) optimization of production of the required cell type for transplantation; (ii) achieving the most favorable delivery of the cells to the subretinal space; and (iii) assessing whether the donor cells are functional and their ability to restore vision. For effective photoreceptor transplantation, the requirement is to achieve synaptic connection of new functional photoreceptors with the host inner nuclear layer (INL) cells. For RPE transplantation, the requirement is to achieve a new polarized RPE monolayer that functions properly to support the photoreceptors. This review provides an update on the recent progress toward restoring vision loss due to retinal degenerative diseases using stem cell-based transplantations and highlights research needed to accelerate clinical progress.

\subsection{Retinal Pigmented Epithelium}

RPE derivation from ESCs and iPSCs has been established by many laboratories over the last decade (Osakada et al. 2008). The recent focus has moved toward the generation of highly homogeneous RPE cultures, with sufficient yield and quality, for clinical use, as well as the evaluation of the best type of cell preparation for delivery to the subretinal space. Different studies have examined the delivery of RPE cells in animal models such as the Royal College of Surgeons rat model of RPE dysfunction. RPE cells can be transplanted into the subretinal space, either as cell suspensions or on different substrates that mimic the Bruch's membrane, i.e., selforganized sheets of cells or biomaterial-based patches (Kamao et al. 2014; Ramsden et al. 2013). Some preservation of the outer nuclear layer (ONL) and visual function has been achieved in animal model studies (Idelson et al. 2009). 
Pre-clinical efforts have led to the setup of clinical trials using RPE derived from human PSCs (Table. 92.1). Several clinical trials have used different types of cell preparation: ESC-RPE cell suspensions (Schwartz et al. 2015), autologous iPSC-RPE sheets (Mandai et al. 2017b), and engineered ESC-RPE patches (Da Cruz et al. 2018; Kashani et al. 2018), for delivery in late-stage AMD and Stargardt's disease. The findings so far indicate no serious adverse effects related to the transplanted cells, with indication of improved visual acuity in some cases. Safety issues regarding potential mutagenesis of patient-derived iPSCs need continued careful evaluation. These initial results warrant further investigation and indicate that treatment of patients at earlier stages of disease may significantly deter its progression.

\subsection{Photoreceptors}

Advances in the ability to differentiate neural retinal cells from human PSCs have provided a platform to generate unlimited numbers of photoreceptors for transplantation (Fig. 92.1). Most significant has been the development of methods to generate in vitro 3D optic cups and the formation of laminated retinal tissue, containing photoreceptors (Nakano et al. 2012; Phillips et al. 2012; Zhong et al. 2014).

One of the outstanding challenges for photoreceptor transplantation therapy is the identification of a delivery system that achieves native-like synaptic connectivity required to restore the lightsensing function to the recipient tissue. Transplantation of photoreceptor precursors (labeled with a GFP transgene) obtained from postnatal mouse retinas, or from mouse ESC cultures, restores the expression of missing outer segment proteins in retinal disease models (Gonzalez-Cordero et al. 2013; Mandai et al. 2017a; Pearson et al. 2012). Transplantation of photoreceptor precursors into late-stage disease models is able to partially reconstruct the degenerated ONL (Singh et al. 
2013). The extensive use of fluorescent transgenes to label donor cell populations has been considered advantageous as a tool to trace fates of transplanted cells in the host tissue. However, recent studies challenged the interpretation that labeled transplanted cells integrate within the host ONL and indicate that a novel mechanism also occurs, whereby donor and host photoreceptor cells exchange material. The transferred material appears to rejuvenate surviving host cells in disease models (Ortin-Martinez et al. 2017; Pearson et al. 2016; Santos-Ferreira et al. 2016). Photoreceptor precursor transplantation thus provides a promising approach to rejuvenate surviving photoreceptors and/or to reconstruct the ONL in late-stage disease.

In recent studies in late-stage disease models which lack an ONL, transplanted human and mouse cones isolated from ESC retinal organoid cultures showed alignment with the INL, as well as expression of mature photoreceptor markers (Gonzalez-Cordero et al. 2017; Kruczek et al. 2017). Retinal sheets made from human ESCs transplanted into primate retinal degeneration models also expressed mature photoreceptor markers. Outer segments were formed in vivo, and there was evidence of some graft synaptic connection with the host INL (Shirai et al. 2015). Nevertheless, evidence that transplanted photoreceptors are functional and act to detect light and transmit visual information to the recipient's brain is limited. Several studies have reported some degree of improved light responses following the transplantation of photoreceptor precursors using a variety of tests. Delivery of mouse primary photoreceptors to a stationary night blindness mouse model provided improved responses to visual cues and visually guided behavior (Pearson et al. 2012). Mouse iPSC progenitors delivered to a mouse end-stage disease model elicited enhanced visual behavior and electroretinogram responses in ex vivo explants (Mandai et al. 2017a). However, modest enhancements in visual behavioral performance were reported after human PSC photoreceptor transplants (Lamba et al. 2009; Barnea-Cramer et al. 2016). 


\subsection{Future Perspectives}

In summary, the last decade has seen impressive advances, as well as setbacks, in the development of stem cell-based treatments to ameliorate or restore vision in retinal degenerative conditions. The large number of clinical trials that are currently in progress for RPE transplantation reflects the leap in development of novel cellular experimental ophthalmological treatments. The significant improvement in gene editing offered by CRISPR/ Cas9 and other nuclease-based editing techniques has opened up avenues to personalized medicine, in which the patient's own cells could be edited to correct harmful mutations in the laboratory and then transferred back into the patient to ameliorate their condition while avoiding immune rejection. Immediate research applications of PSCs are employing patient-derived retinal organoids, for disease modeling, drug screening, and developmental analyses (Ovando-Roche et al. 2017).

For photoreceptor therapy, several critical considerations need addressing. Cell culture systems need refinement, in order to generate photoreceptors that fulfil the criteria of GMP quality and quantity necessary for clinical transplantation. This includes cell purification protocols (Welby et al. 2017) as well as xeno-free conditions. There is a continued need to robustly demonstrate and enhance the formation of synaptic circuitry between donor and host cells. Most evidence to date relies on immunostaining experiments using synaptic markers between host and donor populations. Hence, it is necessary to refine the proof-of-functional connectivity by means of direct electrophysiological and cell tracing approaches. Improvements in the delivery of cells/tissue to the target area could be achieved by supportive matrices, i.e., scaffolds and hydrogels, and co-transplantation of layers of RPE and neural retina, which more closely resemble the tissue native configuration. 
To evaluate cone photoreceptor replacement for macular dystrophies and therapies for complex diseases such as AMD, more relevant pre-clinical animal model studies are needed. There is a need to elucidate the behavior of transplanted cells within the host environment and fathom the material transfer interactions between donor and host tissues. As more clinical trials are conducted based on the encouraging results to date, it will be important to use standardized outcome measures and well-defined target patient groups. Advances in imaging modalities may help to better visualize and track transplanted cells in patients to evaluate the quality of grafts over time. Finally, there is a continued need for strong regulatory guidance and sound study designs to ensure safety to patients. In conclusion, stem cells and their use for transplantation are a promising therapeutic strategy worthy of further exploration and may in the future become a clinical tool to restore vision in a vast array of patients affected by various retinopathies.

This work was supported by the MRC UK grant MR/M015688/1, Great Ormond Street Hospital Children's Charity (GOSHCC), and the National Institute of Health Research (NIHR).

\section{References}

Bainbridge JWB et al (2015) Long-term effect of gene therapy on Leber's congenital amaurosis. N Engl J Med 372:1887-1897

Barnea-Cramer AO et al (2016) Function of human pluripotent stem cell-derived photoreceptor progenitors in blind mice. Sci Rep 6:1-15

Da Cruz L et al (2018) Phase 1 clinical study of an embryonic stem cell-derived retinal pigment epithelium patch in age-related macular degeneration. Nat Biotechnol 36:328-337

Gonzalez-Cordero A et al (2017) Recapitulation of human retinal development from human pluripotent stem cells generates transplantable populations of cone photoreceptors. Stem 
Cell Rep 9:820-837

Gonzalez-Cordero A et al (2013) Photoreceptor precursors derived from three-dimensional embryonic stem cell cultures integrate and mature within adult degenerate retina. Nat Biotechnol 31:741-747

Idelson M et al (2009) Directed differentiation of human embryonic stem cells into functional retinal pigment epithelium cells. Cell Stem Cell 5:396-408

Kamao H et al (2014) Characterization of human induced pluripotent stem cell-derived retinal pigment epithelium cell sheets aiming for clinical application. Stem Cell Rep 2:205-218

Kashani AH et al (2018) A bioengineered retinal pigment epithelial monolayer for advanced, dryage-related macular degeneration. Sci Transl Med 10:1-10

Kruczek K et al (2017). Differentiation and transplantation of embryonic stem cell-derived cone photoreceptors into a mouse model of end-stage retinal degeneration. Stem Cell Rep $8: 1659-1674$

Lamba DA, Gust J, Reh TA (2009) Transplantation of human embryonic stem cell-derived photoreceptors restores some visual function in Crx-deficient mice. Cell Stem Cell 4:7379

MacLaren RE et al (2006) Retinal repair by transplantation of photoreceptor precursors. Nature 444:203-207

Mandai M et al (2017a) iPSC-derived retina transplants improve vision in rd1 end-stage retinaldegeneration mice. Stem Cell Rep 8:69-83

Mandai M et al (2017b) Autologous induced stem-cell-derived retinal cells for macular degeneration. N Engl J Med 376:1038-1046

Nakano T et al (2012) Self-formation of optic cups and storable stratified neural retina from human ESCs. Cell Stem Cell 10:771-785 
Ortin-Martinez A et al (2017) A reinterpretation of cell transplantation: GFP transfer from donor to host photoreceptors. Stem Cells 35:932-939

Osakada F et al (2008) Toward the generation of rod and cone photoreceptors from mouse, monkey and human embryonic stem cells. Nat Biotechnol 26:215-224

Ovando-Roche P et al (2017) Harnessing the potential of human pluripotent stem cells and gene editing for the treatment of retinal degeneration. Curr Stem Cell Rep 3:112-123

Pearson RA et al (2012) Restoration of vision after transplantation of photoreceptors. Nature 485:99-103

Pearson RA et al (2016) Donor and host photoreceptors engage in material transfer following transplantation of post-mitotic photoreceptor precursors. Nat Comm 7:1-15

Pezzullo L et al (2018) The economic impact of sight loss and blindness in the UK population. BMC Health Services Res 18:1-13

Phillips MJ et al (2012) Blood-derived human iPS cells generate optic vesicle-like structures with the capacity to form retinal laminae and develop synapses. Invest Ophthalmol Vis Sci $53: 2007-2019$

Powell C et al (2016) Zebrafish Muller glia-derived progenitors are multipotent, exhibit proliferative biases and regenerate excess neurons. Scientific Rep 6:1-10

Ramsden CM et al (2013) Stem cells in retinal regeneration: past, present and future. Development 140:2576-2585

Santos-Ferreira TF et al (2016) Retinal transplantation of photoreceptors results in donor-host cytoplasmic exchange. Nat Comm 7:1-7

Schwartz SD et al (2015) Human embryonic stem cell-derived retinal pigment epithelium in patients with age-related macular degeneration and Stargardt's macular dystrophy: follow-up of two open-label phase 1/2 studies. Lancet 385:509-516 
Shirai H et al (2015) Transplantation of human embryonic stem cell-derived retinal tissue in two primate models of retinal degeneration. PNAS 113:E81-E90

Singh MS et al (2013) Reversal of end-stage retinal degeneration and restoration of visual function by photoreceptor transplantation. PNAS 110:1101-1106

Welby E et al (2017) Isolation and comparative transcriptome analysis of human fetal and iPSCderived cone photoreceptor cells. Stem Cell Rep 9:1898-1915

Zhong X et al (2014) Generation of three-dimensional retinal tissue with functional photoreceptors from human iPSCs. Nat Comm 5:1-14 
Table 92.1 Clinical trials for stem cell-based therapies for AMD: first reports

\begin{tabular}{l|l|l|l}
\hline Cell preparation & Condition & Outcome & Publication \\
\hline ESC-RPE & $\begin{array}{l}\text { Stargardt's, atrophic } \\
\text { AMD }\end{array}$ & $\begin{array}{l}\text { Vision-related quality-of-life } \\
\text { measures increased }\end{array}$ & $\begin{array}{l}\text { Schwartz et al. } \\
\text { (2015) }\end{array}$ \\
\hline Autologous iPSC-RPE sheet & AMD (one patient) & $\begin{array}{l}\text { Sheet remained intact, no } \\
\text { change in visual acuity }\end{array}$ & $\begin{array}{l}\text { Mandai et al. } \\
\text { (2017b) }\end{array}$ \\
\hline $\begin{array}{l}\text { ESC-RPE monolayer on } \\
\text { polyester-engineered patch }\end{array}$ & $\begin{array}{l}\text { Acute wet AMD (two } \\
\text { patients) }\end{array}$ & $\begin{array}{l}\text { Successful delivery of patch, } \\
\text { visual acuity gain }\end{array}$ & $\begin{array}{l}\text { Da Cruz et al. } \\
(2018)\end{array}$ \\
\hline $\begin{array}{l}\text { ESC-RPE monolayer on } \\
\text { parylene-engineered patch }\end{array}$ & AMD (five patients) & $\begin{array}{l}\text { Successful engraftment of patch, } \\
\text { visual acuity gain }\end{array}$ & $\begin{array}{l}\text { Kashani et al. } \\
\text { (2018) }\end{array}$ \\
\hline
\end{tabular}




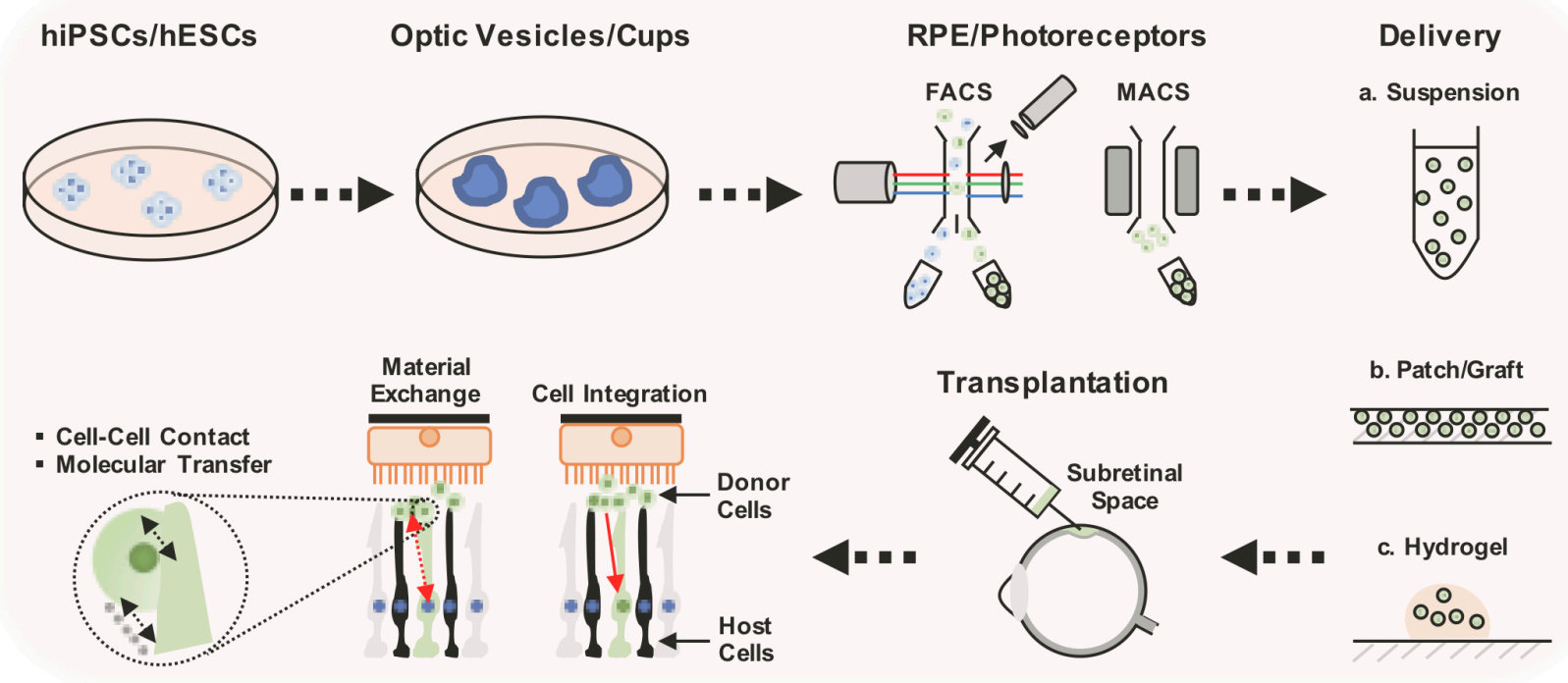

Fig. 92.1 Current approaches for RPE/photoreceptor generation, enrichment, and transplantation. Donor PSCs give rise to RPE/photoreceptors via 3D retinal organoids; cells can be purified using FACS/MACS via cell surface markers or fluorescent labels. Donor cell populations can be delivered to the host in suspension or graft form via subretinal transplantations 